\title{
Package Task Model in Public Packet Mode
}

\author{
Zhuang Rong
}

North China Electric Power University, Baoding, Hebei, China

2695277664@qq.com

\section{Keyword: Crowdsourcing; pricing; Task packing; Reputation value; Multifactor analysis}

\begin{abstract}
In recent years, with the large-scale emergence of mobile Internet applications, enterprises have adopted self-service crowdsourcing platform based on mobile Internet to make business inspection and information collection by using network resources. Combined with the location of multiple tasks in real life, users will choose to compete. Therefore, we should consider the mode of task packing and publishing, and add a new package mode after optimizing the pricing model. This model meets the traffic convenience of the member and the enterprise cost saving target at the same time. The package according to the distance between the individual tasks and play packages should be included within the different pricing tasks, taking into account the mission area member position and reputation value, the number of package will be scheduled task membership quota, multiple factor analysis method, combined with the actual situation, the final model established by MATLAB solution, a pricing model packing mode. Then, the advantages and disadvantages of the model are analyzed and evaluated, and the improvement scheme of the model is also put forward.
\end{abstract}

\section{Problem Background}

In the 2006 issue of the United States journal, the 6-monthly magazine, reporter Jeff Howe first proposed the concept of Crowdsourcing. The so-called crowdsourcing refers to a company or an institution that outsourced the work tasks done by employees in the past in a free and voluntary way to a nonspecific (and usually large) Internet public. With the rise of Internet technology, the development of e-commerce has been booming. At the same time, the emergence of crowdsourcing mode has brought a new way to save costs for enterprises. In the background of the current large data, only by making full use of the network resources, can an enterprise occupy the market as soon as possible. Crowdsourcing mode is to use many active resources on the mobile Internet to solve the traditional way of business such as market research, business inspection, information collection and other tasks. The advantage of this approach is the first to the enterprise, can save cost, with less cost can get a large amount of information; secondly to the society, in this way a great degree of integration of social idle resources, anyone can earn rewards by completing tasks.

In the 2006 issue of the United States journal, the 6-monthly magazine, reporter Jeff Howe first proposed the concept of Crowdsourcing. The so-called crowdsourcing refers to a company or an institution that outsourced the work tasks done by employees in the past in a free and voluntary way to a nonspecific (and usually large) Internet public. With the rise of Internet technology, the development of e-commerce has been booming. At the same time, the emergence of crowdsourcing mode has brought a new way to save costs for enterprises. In the background of the current large data, only by making full use of the network resources, can an enterprise occupy the market as soon as possible. Crowdsourcing mode is to use many active resources on the mobile Internet to solve the traditional way of business such as market research, business inspection, information collection and other tasks. The advantage of this approach is the first to the enterprise, can save cost, with less cost can get a large amount of information; secondly to the society, in this way a great degree of integration of social idle resources, anyone can earn rewards by completing tasks.

Hypothesis:

If a predetermined task limit is only influenced by the membership GPS latitude and longitude and reputation value.

If a member cannot receive a task that is larger than its predetermined limit. 
It is assumed that the pricing of the package task is determined by the pricing of various tasks in the package.

It is assumed that the unit price of a single task within the total price of packaging will decrease with the increase in the number of individual tasks in the package.

Table 1 Symbolic description

\begin{tabular}{cl}
\hline symbol & meanings \\
\hline $\mathrm{X}_{1}$ & The longitude of the task position. \\
$\mathrm{X}_{2}$ & The latitude of the task position. \\
$\mathrm{X}_{3}$ & The distance between the location of the task and the center \\
& point. \\
$\beta_{i}$ & The weight of independent variables in the law of project \\
& pricing. \\
$\alpha_{i}$ & The weight of the independent variable in the new pricing rule \\
& of the project. \\
$G L$ & Membership reservation limit. \\
$n$ & GPS latitude and longitude coordinates of members. \\
$N$ & The number of individual tasks in the package. \\
$\delta$ & Total number of tasks published.
\end{tabular}

\section{Research on the Problem}

In fact, the allocation is based on the proportion of the predetermined quota according to the assignment. According to the data provided by the member position, the member's predetermined task limit and the reputation value, through the analysis of the three indicators of the data, we can see that the membership quota is greatly influenced by the member position and the reputation value. The number of packages will be affected by the predetermined task limit, so we can regard the predetermined task quota as the range of the number of packages, so as to identify the model.

\section{The Establishment of the Model}

By classifying the GPS location of members, we can see that the number of packages will be affected by the quota of members' reservation, and the ML of membership will be affected by the G of the member's position and the $\mathrm{C}$ of reputation value. A model will be built through MATLAB:

$$
M L=\chi G+\varepsilon C+\delta_{3}
$$

The total amount of $\mathrm{P}$ after packaging will be affected by two factors: the number of packages, $\mathrm{N}$, and the total number of N. So, the model expression is:

$$
P_{z}=\sum_{i=1}^{n}\left(\alpha_{i}\left(1-\frac{i}{N}\right) \sum_{j=1}^{3} \beta_{j} X_{j}\right)+\delta
$$

a $(1-\mathrm{i} / \mathrm{N})$ :the contribution rate of the amount to the total amount is expressed when the I task is packaged.

\section{Model Solution}

After clustering, the expression after clustering can be obtained. 


$$
P_{z}=\sum_{i=1}^{n}\left(\alpha_{i}\left(1-\frac{i}{N}\right)\left(-1.11118898 X_{1}+0.098213087 X_{2}+0.003979695 X_{3}\right)\right)+20.05166333
$$

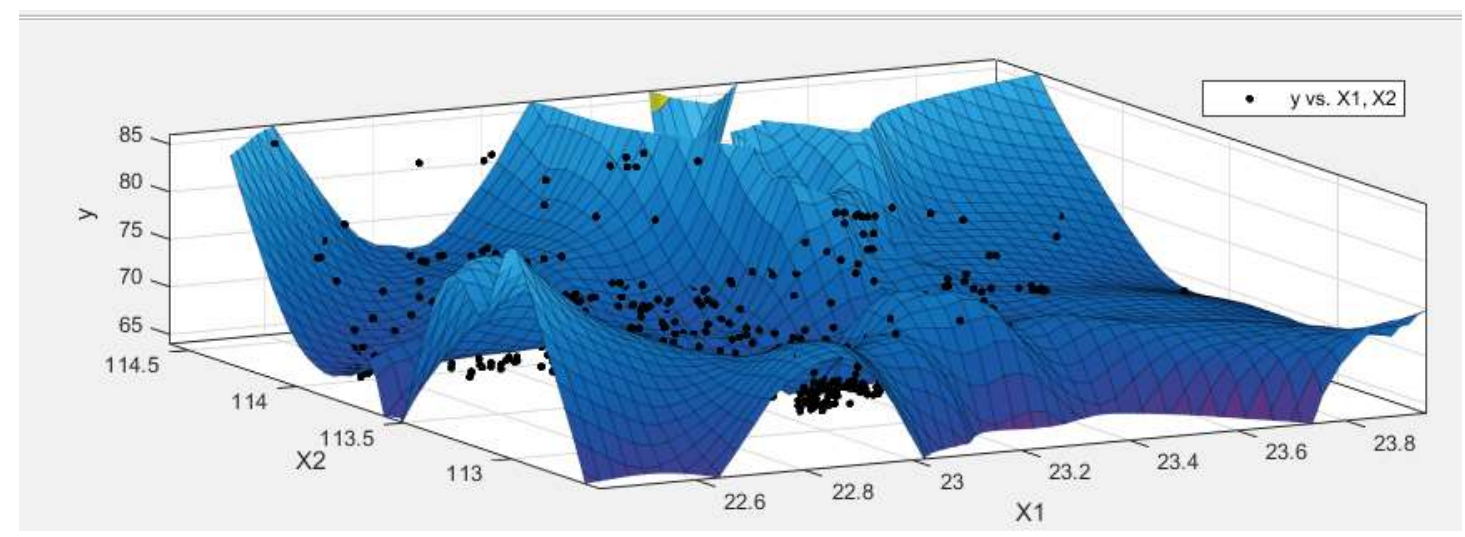

Figure 1. Model after clustering

\section{Model Analysis and Optimization}

Pack release price is not the simple sum of the pricing of individual tasks, but with the increase of the number of individual tasks within the package, the price decline will make a single task $\mathrm{P}$ to package pricing contribution rate; but with the number of single package continued to increase, the same will be made on single task package pricing contribution the rate of. Since the packing tasks will be different tasks together, so the risk of difficult task failure rate will likely shift to risk less difficult task: if there is a particularly difficult task, consider membership may not be completed, the member will because of difficult tasks and give up the whole package all the items together, so simple task completion rate will be reduced, thus increasing the risk of failure from simple tasks; the task of the total, pricing whether science will have a huge impact on the total task, if members feel task returns and pay is not proportional, then, will give up the task of packing member once, give up, give up the equivalent of several individual tasks at the same time, so the task of pricing science will want to pack the completion of the task Great influence.

\section{Advantage of the Model}

This model focuses on the correlation between the data and the physical and chemical indexes, which is beneficial to the simplified model and the reduction of the calculation.

The model is built through many aspects and is committed to dealing with the data with the most concise and clear solution

MATLAB, SPSS, and excel were used for statistics, classification, calculation, and high reliability.

\section{The Limitations of the Model}

Because of the time factor, ignore the impact factor of the proportion of share less, have a certain impact on the accuracy of the model, this paper only makes multiple linear regression, but the linear correlation of the data is not too obvious. It is necessary to make reasonable statistics and classify the data.

\section{The Improvement of the Model}

Some factors can be added, and multiple nonlinear regression equations are considered. Pay attention to the differences between the individual data. In view of the influence of multivariable, factor analysis, principal component analysis, and canonical correlation analysis can be considered. 


\section{Reference}

[1] X.F. Song: Research on behavioral motivation of participants in public package model [D]. Xi'an Electronic and Science University, 2013. E.J. Xia, X.W. Zhao, S. Li: Overseas public package research status and trend $[\mathrm{J}]$. technology economy, 2015, 34 (1): 28-36. (In Chinese)

[2] J. Yan, S.P. Chi, C. Yu: Based on activity of public packet workers reputation model [J]. computer applications, 2017, 37 (7): 2039-2043. (In Chinese)

[3] S. Wang, C. Chen, L. Liang: Analysis of collaborative self organization innovation effect of network public packet model [J]. research management, 2014, 35 (4): 26-33. (In Chinese)

[4] H.Z. Shen: Competency based emergency information management for public package participants selection model research [J]. library science research, 2017 (13): 93-100. (In Chinese)

[5] A.J. An, J. Zhai: Software Crowdsourcing participation factors analysis and forecasting model [J]. computer system application, 2015, 24 (10): 9-16. (In Chinese)

[6] W.W. Ye, L. Zhu: Research on innovation oriented network public packet model characteristics and Implementation Path Research [J]. science research, 2012, 30 (1): 145-151. (In Chinese)

[7] C.Q. Wu, X. Hung: An improved Bias crowdsourcing model based on community discovery [J]. Journal of Xiangtan University, 2015 (4): 87-91. (In Chinese)

[8] S.F. Lin, F. Lin: Public package definition, model research, development and Prospect [J]. science and technology management research, 2015, V.35; No.326 (4): 212-217. (In Chinese)

[9] S. Liu: Research on the application of professional service package model [D]. Beijing University of Technology, 2011. (In Chinese)

[10]H.Q. Han, G.J. Zhao: Research premise and conceptual model construction of G.J. Zhao. Based on the perspective of service leading logic, research on technology management, 2014 (18): 185-191. (In Chinese)

[11]J.H. Fen, G.L. Li, J.H. Feng: A summary of the technical research on crowdsourcing [J]. Journal of computer science, 2015 (9): 1713-1726. (In Chinese) 